\title{
¿Hackearán Nuestro Cerebro? Las Neurotecnologías y el Transhumanismo
}

\section{doi: $10.52749 /$ fh.v2i2.6}

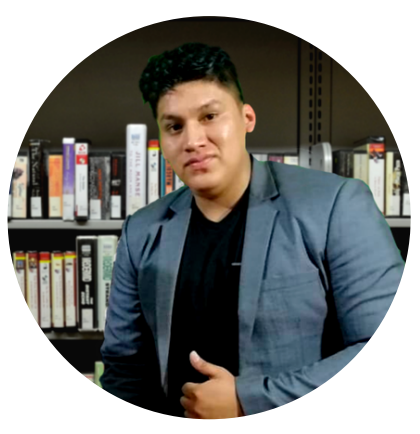

\section{KENNER MORI CASTRO}

https://orcid.org/0000-0002-4749-9166

CEO y cofundador de Innovacit, fundador de la organización social Cultura Steam, divulgador de ciencia, tecnología y educación. Miembro del Consejo Regional de Ciencia, tecnología, emprendimiento e innovación de Huánuco (CORCITEI). Miembro Investigador del Instituto de Estudios Transhumanistas (IET). Formó parte del Consejo Regional de la Juventud - Huánuco. Lic. en Sociología por la Universidad Nacional Hermilio Valdizán, cursa estudios de Maestría en Gestión Pública para el Desarrollo Social e Ingeniería de Sistemas computacionales.

kenner.yesser@gmail.com

(O) @kennermori19

Resumen. El gran avance de las tecnologías digitales y la continua investigación científica han propiciado que el movimiento cultural, filosófico y político del Transhumanismo avance considerablemente. Hoy en día sabemos que los grandes avances que se están dando en cuanto a las tecnologías NBIC (Nanotecnología, biotecnología, infotecnología y cognotecnología) y las Neurotecnologías (Tecnologías que se enfocan en el cerebro y sistema nervioso) son áreas que van a ser claves para el desarrollo superior del transhumanismo. Con estas neurotecnologías ¿podríamos hablar quizás de que alguna empresa, gobierno o grupos de neurohackers podrían invadir nuestro cerebro y hacer modificaciones a nuestros pensamientos, recuerdos y hasta acciones? En este artículo discutimos ciertos peligros de las neurotecnologías y la necesidad de establecer neuroderechos para limitar su uso y consecuencias negativas.

Palabras clave: neurotecnologías, neurohackers, transhumanismo

Desde tiempos remotos el hombre ha intentado superar sus habilidades y reproducir con máquinas actividades de ocio, peligrosas o complejas. Para entender su entorno y crear artefactos, hizo uso, al inicio, del sentido común y, más adelante, de la investigación científica y habilidades técnicas. Esto trajo consigo grandes inventos que hoy usamos a diario, como el televisor, el carro, el reloj, la radio, o algo tan simple como unos lentes para personas con miopía. Un dispositivo de los inventos sin el que no podríamos vivir es el Smartphone o celular inteligente, aparato a donde hemos migrado toda nuestra vida, nuestra personalidad, vida profesional y sentimental y nuestros más íntimos secretos.

Todos dependemos de alguna tecnología que usamos cotidianamente y que no es necesariamente digital, por ejemplo, para preparar alguna comida en un cumpleaños hacemos uso de una cocina, algunas ollas, un cuchillo, un tenedor y más utensilios que encontramos en casa. Para movilizarnos hacia el trabajo, usamos el transporte en bus, moto o manejamos nuestro auto; partimos de esos ejemplos para mencionar que en cierta manera podríamos considerar hasta una pre-etapa del Transhumanismo a la dependencia que tenemos a alguna tecnología externa a nosotros y que la gran mayoría de personas usa en su vida diaria.

\section{¿Qué es el transhumanismo?}

El Transhumanismo podría definirse como un movimiento intelectual, filosófico, cultural y político que busca la mejora del hombre por medio del uso de la tecnología. Por ejemplo, tiene como objetivos que las personas puedan vivir más años, ser inmunes a enfermedades, se conecten a internet, mejoren sus sentidos y fuerza y hasta trasladen su cerebro o consciencia a cuerpos robóticos o a realidades digitales.

En esta Cuarta Revolución Industrial las grandes compañías, también conocidas como las Big Tech, nos traen muchos dispositivos tecnológicos, inteligentes y avanzados. Estas novedades forman parte de lo que la Fundación de Ciencia y Tecnología de EE.UU nombró como tecnologías NBIC (nanotecnología, bio-tecnología, info-tecnología y cognotecnología); un área de desarrollo que va a ser clave para la consecución futura del transhumanismo.

\section{Las Neurotecnologías y el Transhumanismo}

En el marco de las tecnologías NBIC se ha propiciado un gran avance de las Neurotecnologías 
(tecnologías enfocadas en el cerebro y sistema nervioso), las cuales podrían beneficiar de manera considerable a las personas con discapacidad, personas con problemas de visión, problemas de memoria o incluso a aquellas que hayan perdido alguna extremidad y/o se encuentren parapléjicas; sin embargo, es aquí donde se pone en debate si su uso y limitado acceso podrían propiciar más desigualdades sociales y económicas en un mundo tan desemejante como en el que vivimos.

Por otro lado, existe un temor incesante respecto al uso bélico y de dominio que le darían algunos gobiernos con modelos políticos dictatoriales como China, Corea del Norte y otros. Estos podrían crear humanos con habilidades mejoradas, sin considerar los posibles usos en animales, con intereses poco pacíficos. Al igual que los superhéroes de algunos cómics o series de Netflix, como Black Mirror, hoy en día ya se pueden modificar genéticamente animales y se pretenden hacer pruebas en humanos más adelante. Todo esto de la mano de la tecnología CRISPR CAS9. Estamos, entonces, ante el origen de una tecnología de modificación genética que, combinada con las neurotecnologías y otros grandes avances de la IV RI, podría provocar el nacimiento de un humano con habilidades mejoradas y nuevas. De hecho, ya existen personas viviendo con implantes y tecnologías adheridas a su cuerpo, personas que viven con marcapasos cardíacos, riñones o algún órgano impreso en 3D, extremidades robóticas y hasta antenas en el cerebro (como es el caso de NeilHarbinsson, músico $\mathrm{y}$ artista que tiene una antena en el cerebro que le da la capacidad de oír los colores). Estas tecnologías agregadas a un cuerpo biológico nos invitan a pensar que muchas personas puedan no ser definibles como completamente humanas, al menos en términos biológicos, pues requieren de la ayuda de una tecnología para sobrevivir o tener una vida digna. Ante esto nos preguntamos: ¿Podríamos considerarlos ya transhumanos?

\section{¿Hackearán nuestro cerebro?}

La decodificación de toda la actividad cerebral conjuntamente con los adelantos en Inteligencia artificial y Neurotecnologías nos conducen a preguntarnos si en un futuro nuestro cerebro podría ser hackeado. Neuralink, del empresario Elon Musk, es un ejemplo claro del avance tecnológico en este rubro. Su iniciativa ha empezado a desarrollar chips que se puedan conectar con los cerebros humanos $y$, de esa manera, ayudar al tratamiento de personas con parálisis; también se idea su uso para que los humanos nos conectemos a internet y expandamos nuestras habilidades. Esto ya viene probándose en animales como monos y cerdos $y$, en un futuro, se proyectan a hacer pruebas en humanos. Pero Neuralink no es la única, sino que existen otras empresas y dispositivos en el mercado que buscan y permiten Brain-computer interfaces (Interfaz-cerebro computadora), ya sea para controlar drones con la mente (incluso se hacen carreras de este tipo), controlar tu ordenador o hasta controlar tu juego favorito.

¿Te imaginas descargar toda la información de Wikipedia o cualquiera otra plataforma en tu cerebro en poco tiempo? ¿Controlar cualquier dispositivo tecnológico con tu mente e incluso comunicarte telepáticamente?

Con un chip dentro de nuestro cerebro podremos tener la capacidad de controlar otras tecnologías y mover prótesis más avanzadas, acceder a internet, mejorar nuestros sentidos como la visión, habla, entre otros, e incluso de comunicarnos telepáticamente. Sin embargo, ¿podrían algunos neurohackers también tener acceso a nuestros recuerdos, pensamientos y hasta acciones? No es descabellado pensar que tu cerebro pueda ser hackeado por otras personas, empresas e incluso gobiernos con políticas dictatoriales e intenciones mafiosas. Si bien es cierto que para llegar a ese nivel de las neurotecnologías aún falta mucho y de momento solo es un pronóstico futurista, no obstante, no debemos cerrar las puertas a que en algún momento de desarrollo de interfaces cerebro-computador alguien pueda controlar nuestros pensamientos, lo que vemos y quizás muchas de nuestras acciones.

Frente a esto existe un debate ético y moral persistente en disciplinas que evalúan el Transhumanismo y las Neurotecnologías. Algunos comentarios a favor de las mejoras del ser humano y otros en contra. En este último proliferan la Iglesia, las personas con posiciones conservadoras y filosofías distintas a la llustrada, humanista o materialista.

\section{Los neuroderechos}

¿Cómo podríamos regular el Transhumanismo al tener personas mejoradas con tecnología avanzada en su cuerpo?

Rafael Yuste (2020), Neurobiólogo e investigador principal del proyecto BRAIN (por sus siglas en español Investigación del Cerebro a través del Avance de Neurotecnologías Innovadoras), pone énfasis en la necesidad de anticiparse a ciertos ries- 
gos y propone que se pueda tener una legislación específica en cuanto al uso e impacto de las neurotecnologías. En otras palabras, debemos desarrollar Neuroderechos para proteger a los usuarios del uso incorrecto de la tecnología neural.

Asimismo, Marcello Lenca y Roberto Andoro (2017) hacen mención de 04 neuroderechos que debemos considerar. Primero, el derecho a la libertad cognitiva, donde se garantiza a todas las personas tener el poder de decidir con libertad el uso de una nueva interfaz cerebro-máquina. Luego, el derecho a la privacidad mental, con este derecho nosotros podremos elegir cuándo compartir datos neuronales, pero sobre todo bajo qué condiciones. Como punto tres, se hace referencia a la integridad mental, con esto se busca la protección de daños físicos o psicológicos ocasionados a los usuarios por ciertas neurotecnologías. Por último, la continuidad psicológica, derecho que protege nuestra identidad personal en un futuro donde las máquinas puedan tener acceso a ella y alterarla sin nuestro consentimiento previo.

Si bien existen iniciativas como estas para reglamentar y proteger los límites de las neurotecnologías, su uso y avance va a generar una nueva forma de discriminación y desigualdad social. Puesto que, mientras haya empresas con avances tecnológicos que ofrezcan estos dispositivos a las personas, los primeros en adquirirlos serían quienes cuenten con condiciones económicas superiores al promedio. De esa manera se genera una desigualdad mayor entre los que poseen tecnologí- as para mejorar sus habilidades, con los que son personas comunes y no mejoradas, personas sin implantes ni tecnología que les brinden mayores habilidades. Incluso podríamos asemejar esa desigualdad a la brecha tecnológica que se vive hoy en día, específicamente entre los que tienen acceso a internet, a una laptop o un celular y los que no.

En dicho futuro nuestra sociedad estaría dividida entre transhumanos y humanos sin mejoras tecnológicas. Imagine competir con un policía cuya visión incluya rayos $X$ o con un profesional que pueda descargar cualquier información en segundos a su memoria. ¿Podremos competir con cybors o humanos sin límites para sus habilidades físicas y su coeficiente intelectual?

El futuro es incierto, pero muchos de los grandes avances tecnológicos que se tienen hoy en día nos encaminan hacia pronósticos como los expuestos. Imaginar un futuro donde nuestros hijos o nietos puedan dejar de ser humanos completamente $y$ tener partes robóticas para mejorar su calidad de vida es sorprendente, pero al mismo tiempo atemorizante, porque en un mundo lleno de desigualdades en el que el Estado es el que provee subvenciones a los más necesitados, con altos índices de corrupción y donde la delincuencia crece a la par que la tecnología lo hace, no es absurdo imaginar que podríamos ser marionetas vivientes con implantes en el cerebro de neurohackers, gobiernos o empresas. Es imprescindible un análisis ético y moral sobre todo esto ipsofacto.

\section{Bibliografía}

Martín, B. (24 de marzo de 2020). ¿Estamos preparados para la llegada del 'neurocapitalismo'? BBVAOpenMind: https://www.bbvaopenmind.com/tecnologia/innovacion/estamospreparados-llegada-del-neurocapitalismol

Lenca, M., Andorno, R. Towards new human rights in the age of neuroscience and neurotechnology. Life Sci Soc Policy 13, (5) (2017). https://doi.org/10.1186/s40504-017-0050-1

\section{Cómo citar este artículo:}

Mori, K. (2021). ¿Hackearán Nuestro Cerebro? Las Neurotecnologías y el Transhumanismo. Futuro Hoy, 2(2), 3537. https://doi.org/10.52749/fh.v2i2.6

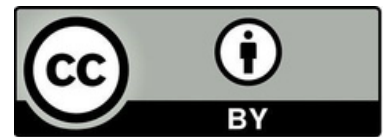

Esta obra está bajo licencia internacional Creative Commons 4.0 Reconocimiento 4.0. 